\title{
Signal Sparse Decomposition Based on Adaptive Chaos Fruit Fly Optimization Algorithm
}

\author{
Ming Yang ${ }^{1,}$, , Wei Liu ${ }^{1, b^{*}}$, Beichen Chen ${ }^{1}$ \\ ${ }^{1}$ College of Information and Control Engineering, Jilin Institute of Chemical Technology, \\ Jilin 132022, Jilin China \\ aymdjx@126.com, byangm521@126.com \\ * corresponding author
}

\begin{abstract}
Keywords: Sparse decomposition; Adaptive Chaos Fruit Fly Optimization Algorithm(ACFOA); Global optimum; Computational complexity

Abstract. Sparse decomposition can represent signal with small number of atoms. But, its high computation complexity hinders practical application. Fruit Fly Optimization Algorithm (FOA) improves the efficiency of atoms' searching, but the solution sometimes is not global optimum. In order to solve this problem, Adaptive Chaos Fruit Fly Optimization Algorithm (ACFOA) is presented. In this paper, signal sparse decomposition based on ACFOA is presented. The experiment shows that the reconstructed signal is satisfied, and the computational complexity is reduced greatly
\end{abstract}

\section{Introduction}

In the process of signal processing and analysis, how to use fewer components to represent the original signal is a very important research topic. The traditional signal decomposition method is mainly orthogonal decomposition, such as fourier transform and cosine transform. The signal is decomposed in a set of complete orthogonal basis. But due to the orthogonality of the base, the decomposition is usually unsparse. This limits the application range of signal decomposition [1]. Therefore, in order to get the sparse representation of signal, decomposition basis must be expanded. And then, the complete orthogonal basis converts to over-complete redundant dictionary, and this is sparse decomposition. Sparse decomposition need to search out the most proximal atom with the decomposed signal from the over-complete dictionary, so the process of decomposition is very complex.

Reducing the computational complexity of sparse decomposition, the key is to find more concise, more rapid method to search optimal atom. Intelligent algorithm has global optimization performance, and it is suitable for parallel processing. Apply it to the optimal atom search process of sparse decomposition, the search efficiency will be improved greatly. At present, the genetic algorithm [2], fish swarm algorithm [3], particle swarm algorithm [4], and other intelligent algorithms have been widely used in sparse decomposition. Fruit Fly Optimization Algorithm (FOA) is a new intelligent algorithm. Compared with other intelligent algorithms, the algorithm is simple, easy to understand and implement. But FOA is easy to fall in local optimum [5]. In order to solve this problem, references [6] proposed an improved algorithm - adaptive chaotic fruit fly optimization algorithm(ACFOA). If the FOA is local optimum, chaotic algorithm will be used adaptively to search global optimum. In this paper, signal sparse decomposition based on ACFOA is presented.

\section{Adaptive Chaos Fruit Fly Optimization Algorithm(ACFOA)}

ACFOA is presented based on FOA. If FOA is trapped in local optimum, chaos algorithm will be used. ACFOA operation process is as follows [6]:

(1)Set parameters: maximum number of iterations Maxgen, fruit fly swarm size SizePop, random initialized location of random fruit fly swarm Init $X_{-}$axis, Init $Y_{-}$axis, flavour intensity variance threshold $\delta$, the number of ergodic-times $M$;

(2)Given the random direction and distance of fruit fly individuals foraging: 


$$
\left\{\begin{array}{c}
X_{i}=X_{-} \text {axis }+ \text { Random Value } \\
Y_{i}=Y_{-} \text {axis }+ \text { Random Value }
\end{array}\right.
$$

(3)As the food location is unknown, estimate the distance from the origin, and calculate the flavour intensity of next position:

$$
\begin{aligned}
& \text { Dist }_{i}=\sqrt{X_{i}^{2}+Y_{i}^{2}} \\
& S_{i}=1 / \text { Dist }_{i}
\end{aligned}
$$

(4)Calculate the flavour intensity of fruit fly individuals $\mathrm{Smell}_{i}$ :

$$
\text { Smell }_{i}=\text { Function }\left(S_{i}\right)
$$

(5)Calculate the best flavour intensity of fruit fly swarm:

$$
[\text { best } \text { Smell bestindex }]=\max \left(\text { Smell }_{i}\right)
$$

(6)Record coordinates of the best flavour intensity, fruit fly swarm fly to the coordinates:

$$
\begin{gathered}
\text { Smellbest }=\text { bestSmell } \\
X_{-} \text {axis }=X(\text { bestindex }) \\
Y_{-} \text {axis }=Y(\text { bestindex })
\end{gathered}
$$

(7)Calculate the average and variance of fruit fly swarm flavour intensities:

$$
\begin{aligned}
& \text { Smell }_{\text {avg }}=\sum_{i=1}^{\text {SizePop }_{\text {Smell }} / \text { SizePop }} \\
& \sigma^{2}=\sum_{i=1}^{\text {SizePop }}\left(\text { Smell }_{i}-\text { Smell }_{\text {avg }}\right)^{2}
\end{aligned}
$$

(8)If $\sigma^{2}<\delta$ and $M>0$, map $X_{i}$ and $Y_{i}$ to chaotic variables $C X_{i}$ and $C Y_{i}$ according to the Eq. 9 and the logistic mapping. And then, transform $C X_{i}$ and $C Y_{i}$ according to the Eq. 10. Finally, get new coordinates $\left(X_{i}^{\prime}, Y_{i}^{\prime}\right)$ with Eq. 11; at the same time, $M=M-1$; Otherwise, skip to step (11);

$$
\begin{aligned}
& \left\{\begin{array}{cc}
C X_{i}=\left(X_{i}-a_{i}\right) /\left(b_{i}-a_{i}\right) & X_{i} \in\left[a_{i}, b_{i}\right] \\
C Y_{i}=\left(Y_{i}-c_{i}\right) /\left(d_{i}-c_{i}\right) & Y_{i} \in\left[c_{i}, d_{i}\right]
\end{array}\right. \\
& \left\{\begin{array}{l}
C X(t+1)_{i}=4 C X(t)_{i}\left(1-C X(t)_{i}\right) \\
C Y(t+1)_{i}=4 C Y(t)_{i}\left(1-C Y(t)_{i}\right)
\end{array} \quad t \in[1, M]\right. \\
& \left\{\begin{array}{c}
X_{i}^{\prime}=a_{i}+C X_{i}\left(b_{i}-a_{i}\right) \\
Y_{i}^{\prime}=c_{i}+C Y_{i}\left(d_{i}-c_{i}\right)
\end{array}\right.
\end{aligned}
$$

(9)Estimate the distance between the new coordinates $\left(X_{i}^{\prime}, Y_{i}^{\prime}\right)$ and the origin, and calculate the flavour intensity of the position: 


$$
\begin{aligned}
& \text { Dist }_{i}^{\prime}=\sqrt{X_{i}^{\prime 2}+Y_{i}^{\prime 2}} \\
& S_{i}^{\prime}=1 / \text { Dist }_{i}^{\prime} \\
& \text { Smell }_{i}^{\prime}=\text { Function }\left(S_{i}^{\prime}\right)
\end{aligned}
$$

(10) If Smell ${ }_{i}^{\prime}>$ Smellbest, Smellbest $=$ Smell $_{i}^{\prime}, X_{-}$axis $=X_{i}^{\prime}, Y_{-}$axis $=Y_{i}^{\prime}$, then go to step (8); Otherwise, go to step (8) directly;

(11) Repeat step (2) - (10), until reach the maximum number of iterations.

\section{Signal Sparse Decomposition Based on ACFOA}

The basic principle of sparse decomposition is to find an approximate linear combination with a small number of atoms to represent original signal, expressed with Eq. 15.

$$
f \approx \sum_{k=0}^{n-1}\left\langle R^{k} f, g_{\gamma_{k}}\right\rangle g_{\gamma_{k}}
$$

Among them, $g_{\gamma_{k}}$ is the atom of over-complete dictionary, $\left\langle R^{k} f, g_{\gamma_{k}}\right\rangle$ is the projection on $g_{\gamma_{k}}$ of original signal and its decomposition residual. In fact, every atom searching in sparse decomposition is to find the maximum of $\left\langle R^{k} f, g_{\gamma_{k}}\right\rangle$. So, searching each atom need to calculate the inner product of original signal or its decomposition residual with each atom in over-complete dictionary. So, one way to reduce computational complexity is to reduce the calculation amount of searching optimal atoms.

Because FOA sometimes fall in local optimum, this paper uses ACFOA in signal sparse decomposition. Chaos algorithm is not carry out inner product operation, so ACFOA relative to FOA does not increase the computational complexity. In our algorithm, atom dictionary is Gabor:

$$
g_{\gamma}=\frac{1}{\sqrt{s}} g\left(\frac{t-u}{s}\right) \cos (v t+w)
$$

Among them, $g(t)=e^{-s t^{2}}$ is the Gaussian window function, $\gamma=(s, u, v, w)$ is the time and frequency parameters of $g(t)$. By the laws of dictionary discretization, if the length of signal is $L$, atom number of Gabor dictionary is $L_{D}=52\left(L \log _{2} L+L-1\right)$ [7] .So, the length of signal is 256, the size of atom dictionary which is used in sparse decomposition is $L_{D}=119756$. ACFOA algorithm is applied to the signal sparse decomposition, and the corresponding parameters $\gamma=(s, u, v, w)$ are transferred into four dimensional searching spaces of ACFOA. Also, for the 256 long signal, the range of $s$ and $u$ is $[1,256]$, the range of $v$ and $w$ is $[0,2 \pi]$. Set the fruit fly population size SizePop $=50$, the maximum number of iterations Maxgen $=80$, the inner product times of each atom in the process of decomposition is 4000. Compared with traditional sparse decomposition, the number of inner product is reduced greatly. Because FOA may be trapped in local optimum, so ACFOA is applied in this article to the signal sparse decomposition. When FOA is not the global optimal, chaotic solution is used to overcome local optimal.

\section{Experiment}

To compare related experimental results, the experiment signal and experiment conditions are similar to reference [7], specific as follows: the fruit fly population size SizePop $=50$, the number of 
iterations Maxgen $=80$, the number of ergodic-times $M=5$, flavor concentration variance threshold $\delta=0.01$, signal length 256, decomposition atom number 30. Experimental results are shown in Fig. 1 . The corresponding mean square error of FOA and ACFOA is 44.73 and 43.02. It is shown that the reconstructed effect of ACFOA is better than FOA. The main reason is the intelligent algorithm will inevitably fall into local optimum. This paper has carried on the chaos optimization to local optimal adaptively, and the result is more accurate.

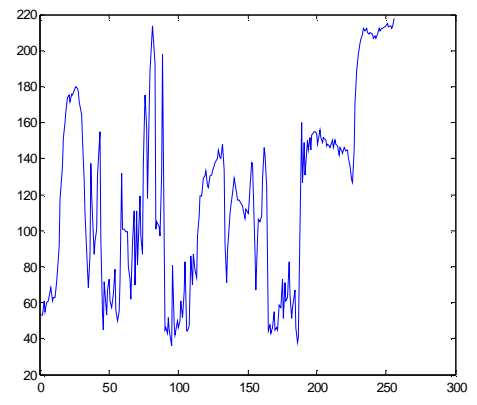

(a) Original signal

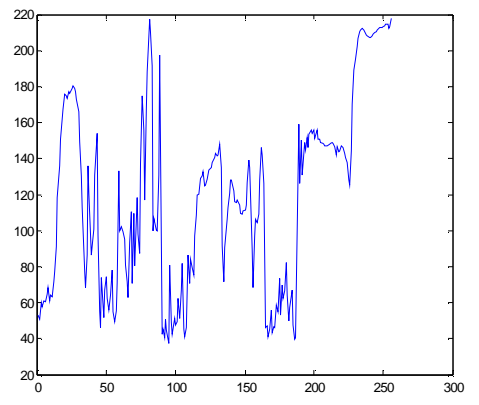

(b)FOA

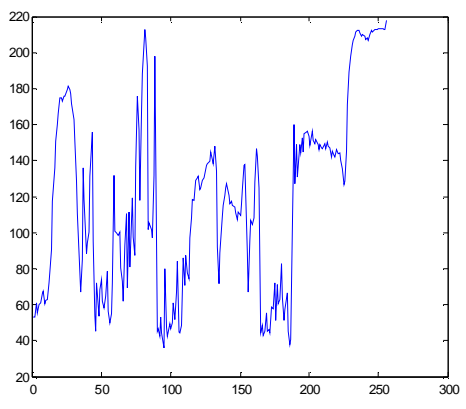

(c)ACFOA

Fig. 1. Experiment signal and sparse decomposition reconstructed signals with different algorithms

\section{Conclusions}

Because of the great calculation amount of traditional sparse decomposition, decomposition is so slow that hinders its application. Intelligent algorithm has good performance in parallel computing. Apply it to search the optimal atom in sparse decomposition, the number of inner product is reduced greatly. As a new intelligent algorithm, FOA algorithm is simple and easy to understand. ACFOA, improved based on FOA, overcome the local optimum adaptively. This paper applies ACFOA in signal sparse decomposition, and the result is satisfied.

\section{References}

[1] Hua ZHANG, Quantum-inspired Evolutionary Algorithm and Its Application in Image Sparse Decomposition, Chengdu: Southwest Jiaotong University, pp. 11-15, 2010.

[2] Chunjuan OUYANG, Xia LI, Bin LI, A Steganographic Algorithm Based on Image Sparse Decomposition Optimized by GA. Signal Processing, 28(6), pp. 821-826, 2012.

[3] Ailing QI, Hongwei MA, Tao LIU, Ultrasonic Signal Sparse Decomposition Based on Improved Artificial Fish Swarm Optimization Algorithm. Chinese Journal of Scientific Instrument, 30(12), pp. 2480-2484, 2009.

[4] Hengjian LI, Zhongke YIN, Jiashu ZHANG, Jianying WANG, Image Sparse Decomposition Based on Particle Swarm Optimization with Chaotic Mutation. Journal of Southwest Jiaotong University, 43(4), pp. 509-513, 2008.

[5] Hui CHENG, Chengzhong LIU, Mixed Fruit Fly Optimization Algorithm Based on Chaotic Mapping. Computer Engineering, 39(5), pp. 218-221, 2013.

[6] Junying HAN, Chengzhong LIU, Adaptive Chaos Fruit Fly Optimization Algorithm. Journal of Computer Application, 33(5), pp. 1313-1316, 2013.

[7] Zheng'an XIAO, Speech Signal Sparse Decomposition with FOA. Computer Engineer and Applications, 49(10), pp. 232-234, 2013. 